\title{
Unstable gravitino dark matter and neutrino flux
}

\author{
Laura Covi, ${ }^{a}$ Michael Grefe, ${ }^{a}$ Alejandro Ibarra $^{b}$ and David $\operatorname{Tran}^{b}$ \\ ${ }^{a}$ Deutsches Elektronen-Synchrotron DESY, Hamburg, \\ Notkestraße 85, 22603 Hamburg, Germany \\ ${ }^{b}$ Physik-Department T30, Technische Universität München, \\ James-Franck-Straße, 85748 Garching, Germany \\ E-mail: laura.covi@desy.de, michael.grefe@desy.de, alejandro.ibarra@ph.tum.de, \\ david.tran@ph.tum.de
}

Received October 20, 2008

Revised December 9, 2008

Accepted December 10, 2008

Published January 15, 2009

\begin{abstract}
The gravitino is a promising supersymmetric dark matter candidate which does not require exact $R$-parity conservation. In fact, even with some small $R$-parity breaking, gravitinos are sufficiently long-lived to constitute the dark matter of the Universe, while yielding a cosmological scenario consistent with primordial nucleosynthesis and the high reheating temperature required for thermal leptogenesis. In this paper, we compute the neutrino flux from direct gravitino decay and gauge boson fragmentation in a simple scenario with bilinear $R$-parity breaking. Our choice of parameters is motivated by a proposed interpretation of anomalies in the extragalactic gamma-ray spectrum and the positron fraction in terms of gravitino dark matter decay. We find that the generated neutrino flux is compatible with present measurements. We also discuss the possibility of detecting these neutrinos in present and future experiments and conclude that it is a challenging task. However, if detected, this distinctive signal might bring significant support to the scenario of gravitinos as decaying dark matter.
\end{abstract}

Keywords: dark matter, neutrino and gamma astronomy, cosmology of theories beyond the $\mathrm{SM}$

ArXiv ePrint: 0809.5030 


\section{Contents}

1 Introduction 1

2 Bilinear $R$-parity breaking $\quad 2$

2.1 Gravitino decay 4

3 Neutrino energy spectrum 5

$\begin{array}{lll}4 & \text { Neutrino fluxes } & 7\end{array}$

5 Neutrino backgrounds $\quad 10$

6 Detection prospects $\quad 11$

6.1 Comparison with the signal 11

6.2 Electron and muon neutrinos 12

6.3 Tau neutrinos 13

6.4 Observability in Super-Kamiokande 13

7 Future detectors $\quad \mathbf{1 5}$

7.1 Hyper-Kamiokande 16

$\begin{array}{ll}7.2 & \text { IceCube and } \mathrm{km}^{3} \text { detectors } \\ \end{array}$

8 Conclusions $\quad 16$

\section{Introduction}

The question of the nature of dark matter is still one of the unsolved mysteries in modern cosmology. Many particle candidates have been put forward, but until now the only dark matter evidence we have is based on the gravitational interaction. In the context of supersymmetry with conserved $R$-parity, one naturally encounters one of the most favoured solutions to the dark matter problem. There, the lightest supersymmetric particle (LSP) is stable and can be a successful dark matter candidate if it is neutral and weakly interacting like the neutralino. The neutralino is the most thoroughly studied dark matter candidate and will be tested in the near future in accelerator, direct detection and indirect detection experiments [1].

On the other hand, it is also possible that the dark matter interacts only gravitationally, and supersymmetry also offers candidates of this type. A prominent example is the gravitino, the superpartner of the graviton, which was the first supersymmetric dark matter candidate proposed [2]. It is one of the most elusive dark matter candidates due to its extremely weak interactions. In fact, as part of the gravity multiplet, all gravitino interactions are suppressed either by the Planck scale (for the spin-3/2 component) or by the supersymmetry-breaking scale (for the Goldstino component).

Usually, having an LSP with such extremely weak interactions poses a severe problem to Big Bang nucleosynthesis, as it makes the next-to-lightest supersymmetric particle (NLSP) so long-lived that it decays during or after the formation of the primordial nuclei, typically 
spoiling the successful predictions of the standard scenario [3]. Moreover, if the NLSP is charged, the formation of a bound state with ${ }^{4} \mathrm{He}$ catalyzes the production of ${ }^{6} \mathrm{Li}[4]$ leading to an overproduction of ${ }^{6} \mathrm{Li}$ by a factor 300-600 [5]. One way to avoid these constraints is to lower the scale of supersymmetry breaking, thus enhancing the Goldstino interactions. However, the reheating temperature of the Universe has to be lowered accordingly in order to avoid overclosure [6] and is then in conflict with the minimal value required by thermal leptogenesis in order to explain the baryon asymmetry of the Universe [7].

It was recently proposed in [8] that these problems are automatically solved if a small breaking of $R$-parity is introduced in the model. Even though in the presence of $R$-parity violation the neutralino LSP is too short-lived to play the role of dark matter, the gravitino LSP can still have a sufficiently long lifetime, which is typically many orders of magnitude greater than the age of the Universe due to the suppression of the decay rate by the Planck scale and the small $R$-parity violating couplings [9]. In this scenario, the NLSP population in the early Universe quickly decays into Standard Model particles via $R$-parity violating interactions. Apart from the presence of a rather inert population of gravitinos, produced thermally from superpartner scatterings at reheating, the cosmology then reduces to the non-supersymmetric case well before the synthesis of primordial nuclei.

An intriguing feature of the scenario with $R$-parity breaking is that gravitino dark matter is not necessarily invisible anymore, since it will decay into Standard Model particles at a very slow rate. Since the huge number of gravitinos in our own Galaxy, as well as in nearby galaxies and clusters, may compensate for the highly suppressed decay rate, this opens up the possibility of observing the dark matter decay products as an anomalous contribution to the diffuse gamma-ray flux $[10,11]$ or the cosmic-ray antimatter fluxes $[12,13]$. Indeed, anomalous excesses have been observed both in the diffuse extragalactic gamma-ray spectrum and in the positron fraction in similar energy ranges. It has been pointed out that the decay of gravitino dark matter with a lifetime of $\sim 10^{26} \mathrm{~s}$ and a mass of $\sim 150 \mathrm{GeV}$ can account for both of these excesses at the same time $[12,13]$. This motivates our study of the corresponding neutrino spectrum for the same choice of parameters, both as a consistency check and to find out whether in this scenario an anomalous contribution to the neutrino flux may be expected in present and future neutrino experiments.

This paper is organised as follows: in the next section we will briefly review bilinear $R$ parity violating models and discuss the resulting gravitino decay modes. In section 3 we will present the neutrino spectrum from dark matter decay and describe its main features. In section 4 we will then give the neutrino flux as a function of the gravitino lifetime and mass both for neutrinos from our own Galaxy and from diffuse extragalactic sources and consider the effect of neutrino oscillations on the signal expected at the Earth. In section 5 we will discuss the different neutrino backgrounds in the energy range we are interested in and compare them to our signal. In section 6 we will propose strategies to disentangle the signal from the background, compare the result to present neutrino data from Super-Kamiokande. We will then discuss the feasibility of detection in future detectors in section 7 and conclude in section 8 .

\section{Bilinear $R$-parity breaking}

We consider here a model of the type described in detail in [8], where the breaking of $R$-parity is related to the breaking of $B-L$. In this class of models, the lepton number violation in the superpotential is encoded in the bilinear term [14]

$$
W_{\not R p}=\mu_{i} L_{i} H_{u} .
$$


It can be shown that the $R$-parity violating coupling $\mu_{i}$ is suppressed compared to the coefficient $\mu$ of the $R$-parity conserving bilinear term $H_{u} H_{d}$ by $\mu_{i} \sim \mu v_{B-L}^{2} / M_{P}^{2}$, with $v_{B-L}$ and $M_{P}$ being the scale of $B-L$ breaking and the Planck mass, respectively. On the other hand, the induced baryon number violation is negligible, the corresponding Yukawa coupling being further suppressed by the ratio of the gravitino mass to the Planck mass and a higher power of the scale of $B-L$ breaking over the Planck scale, $\lambda^{\prime \prime} \sim m_{3 / 2} v_{B-L}^{4} / M_{P}^{5}$. Then, if the scale of $B-L$ breaking is low enough, the constraints on proton stability are satisfied and the lepton number violating interactions are kept out of equilibrium before the electroweak phase transition, thus preventing the erasure of any previously generated baryon asymmetry. This condition requires approximately $\mu_{i} / \mu \lesssim 10^{-6-7}$ [15], which translates into $v_{B-L} \lesssim 10^{14} \mathrm{GeV}$, although this could be circumvented for some specific flavour structures in the Yukawa couplings. Since the present bounds on the gravitino lifetime are already constraining the parameters at this order from observations of both gamma rays $[10,11]$ and antiprotons $[12,13]$, this last option is probably not viable.

Apart from the supersymmetric term above, the corresponding soft bilinear supersymmetry-breaking term $B_{i} \mu_{i} \widetilde{L}_{i} H_{u}$ arises in the Lagrangian. Since the $B_{i}$ and $\mu_{i}$ terms are not usually aligned at the weak scale, a non-vanishing vacuum expectation value (v.e.v.) is generated along the sneutrino field direction explicitly breaking lepton number and generating not only one neutrino mass, but also non-vanishing mixings between neutralinos and neutrinos, as well as between charginos and charged leptons [16]. Such mixings are responsible for the two-body decays of the gravitino into gauge boson and neutrino, which are the main source of neutrino flux in our scenario. These decays are also possible at the one-loop level if only trilinear $R$-parity breaking terms are considered, and even in this case they can dominate in part of the parameter space [17]. Since the neutralino-neutrino mixing takes place along the Zino component, the branching ratios into the different gauge boson channels are fixed by the neutralino mixing matrix once the gravitino mass is specified.

If the gravitino is lighter than the massive gauge bosons, the dominant decay channel is the two-body decay into monoenergetic photon and neutrino. Since all the observed Yukawa couplings are largest for the third generation, it is reasonable to assume that the $R$-parity breaking couplings are also largest for the third generation. Thus, the sneutrino acquires a v.e.v. only along the $\widetilde{\nu}_{\tau}$ direction, and the gravitino will dominantly decay into neutrinos with $\tau$ flavour. We will see later that this is not a crucial assumption, since neutrino oscillations change any pure neutrino flavour into a mixed state. In particular, due to maximal atmospheric mixing, the flux of tau and muon neutrinos turns out to be identical.

If instead the gravitino is heavier than the electroweak gauge bosons, the decay into these particles is favoured, since the sneutrino has electroweak charge. In this case, neutrinos are produced not only in the direct decay into $Z^{0} \nu_{\tau}$, but also in the fragmentation of the $W^{ \pm}$and $Z^{0}$ bosons and the decay of the $\tau^{\mp}$ leptons, thus adding a continuous component to the spectrum. In this paper, we will mostly consider the second case of a heavier gravitino, motivated by the interpretation of anomalies in other channels as explained above. Furthermore, at lower gravitino masses, the detection of any signal is much more difficult due to the lower neutrino yield and the smaller signal-to-background ratio. 


\subsection{Gravitino decay}

In the models with bilinear $R$-parity breaking and a non-zero sneutrino v.e.v. along the $\widetilde{\nu}_{\tau}$ direction, the main decay channels for the gravitino are:

$$
\begin{aligned}
& \psi_{3 / 2} \rightarrow \gamma \nu_{\tau}, \\
& \psi_{3 / 2} \rightarrow W^{ \pm} \tau^{\mp}, \\
& \psi_{3 / 2} \rightarrow Z^{0} \nu_{\tau}, \\
& \psi_{3 / 2} \rightarrow h \nu_{\tau} .
\end{aligned}
$$

The first decay is practically always allowed, while the next two are open only for a gravitino mass above the threshold for $W^{ \pm}$or $Z^{0}$ production. At even higher masses, the decay into Higgs boson and neutrino opens up via the Higgsino component of the neutralino and the $R$ parity violating Higgs-sneutrino mixing. We will consider here the case of a large Higgs mass parameter $\mu$, where the lightest Higgs is Standard Model-like and the other Higgses decouple.

The decay widths for these processes can be computed from the interaction Lagrangian of a gravitino with a gauge boson and a gaugino or with the two chiral Higgs multiplets with the insertion of a sneutrino v.e.v. [18]. The decays into electroweak gauge bosons arise both from 3 -vertices and from the non-abelian 4-vertex, so their structure is more complicated than for the abelian sector. The results depend on the gaugino mixing matrices and are given by the following:

$$
\begin{aligned}
\Gamma\left(\psi_{3 / 2} \rightarrow \gamma \nu_{\tau}\right) & =\frac{\xi_{\tau}^{2} m_{3 / 2}^{3}}{64 \pi M_{P}^{2}}\left|U_{\widetilde{\gamma} \widetilde{Z}}\right|^{2}, \\
\Gamma\left(\psi_{3 / 2} \rightarrow Z^{0} \nu_{\tau}\right) & =\frac{\xi_{\tau}^{2} m_{3 / 2}^{3}}{64 \pi M_{P}^{2}} \beta_{Z}^{2}\left[\left|U_{\widetilde{Z} \widetilde{Z}}\right|^{2} f_{Z}-\frac{8}{3} \frac{M_{Z}}{m_{3 / 2}} \operatorname{Re}\left[U_{\widetilde{Z} \widetilde{Z}}\right] j_{Z}+\frac{1}{6} h_{Z}\right], \\
\Gamma\left(\psi_{3 / 2} \rightarrow W^{ \pm} \tau^{\mp}\right) & =\frac{\xi_{\tau}^{2} m_{3 / 2}^{3}}{32 \pi M_{P}^{2}} \beta_{W}^{2}\left[\left|U_{\widetilde{W} \widetilde{W}}\right|^{2} f_{W}-\frac{8}{3} \frac{M_{W}}{m_{3 / 2}} \operatorname{Re}\left[U_{\widetilde{W} \widetilde{W}}\right] j_{W}+\frac{1}{6} h_{W}\right],
\end{aligned}
$$

with $\xi_{\tau}=\left\langle\widetilde{\nu}_{\tau}\right\rangle / v$, where $v=174 \mathrm{GeV}$ is the Higgs v.e.v.. Assuming $\xi_{\tau} \ll 1$, the mixing matrix elements are given by

$$
\begin{aligned}
& U_{\widetilde{\gamma} \widetilde{Z}} \simeq M_{Z} \sum_{\alpha=1}^{4} \frac{c_{\widetilde{\gamma} \chi_{\alpha}} c_{\widetilde{Z} \chi_{\alpha}}^{*}}{M_{\alpha}} \\
& U_{\widetilde{Z} \widetilde{Z}} \simeq M_{Z} \sum_{\alpha=1}^{4} \frac{c_{\widetilde{Z} \chi_{\alpha}} c_{\widetilde{Z} \chi_{\alpha}}^{*}}{M_{\alpha}}, \\
& U_{\widetilde{W} \widetilde{W}} \simeq \frac{M_{W}}{2} \sum_{\alpha=1}^{2} \frac{c_{\widetilde{W}^{+} \chi_{\alpha}^{+}} c_{\widetilde{W}^{-} \chi_{\alpha}^{-}}^{*}+h . c .}{M_{\alpha}^{ \pm}} .
\end{aligned}
$$

Here, $c_{i j}$ are the elements of the unitary matrices that diagonalise the neutralino/chargino 
mass matrices. The kinematical factors are given by

$$
\begin{aligned}
\beta_{i} & =1-\frac{M_{i}^{2}}{m_{3 / 2}^{2}}, \\
f_{i} & =1+\frac{2}{3} \frac{M_{i}^{2}}{m_{3 / 2}^{2}}+\frac{1}{3} \frac{M_{i}^{4}}{m_{3 / 2}^{4}}, \\
j_{i} & =1+\frac{1}{2} \frac{M_{i}^{2}}{m_{3 / 2}^{2}}, \\
h_{i} & =1+10 \frac{M_{i}^{2}}{m_{3 / 2}^{2}}+\frac{M_{i}^{4}}{m_{3 / 2}^{4}} .
\end{aligned}
$$

The non-abelian 4-vertex computed here was previously neglected in [11]. Note that our results for the decay rates do not agree exactly with those given in [13]: for the interference terms proportional to $j_{i}$ we find a negative sign and a larger coefficient. As a result, the branching ratios into the massive gauge boson channels are slightly smaller than in [13], while the branching ratio into photon-neutrino is larger.

The decay into the lightest Higgs, on the other hand, is given by

$$
\Gamma\left(\psi_{3 / 2} \rightarrow h \nu_{\tau}\right)=\frac{\xi_{\tau}^{2} m_{3 / 2}^{3}}{384 \pi M_{P}^{2}} \beta_{h}^{4}\left|U_{\widetilde{H}_{u} \widetilde{Z}} \sin \beta+U_{\widetilde{H}_{d} \widetilde{Z}} \cos \beta+\frac{m_{\widetilde{\nu}_{\tau}}^{2}}{m_{\widetilde{\nu}_{\tau}}^{2}-m_{h}^{2}}\right|^{2},
$$

in the limit where the lightest Higgs is given by $h=\sqrt{2}\left(\operatorname{Re}\left[H_{u}\right] \sin \beta+\operatorname{Re}\left[H_{d}\right] \cos \beta\right)$, with

$$
U_{\widetilde{H}_{i} \widetilde{Z}} \simeq M_{Z} \sum_{\alpha=1}^{4} \frac{c_{\widetilde{H}_{i} \chi_{\alpha}} c_{\widetilde{Z}_{\chi_{\alpha}}}^{*}}{M_{\alpha}},
$$

and expressing the soft supersymmetry-breaking Higgs-sneutrino mixed mass term through the sneutrino v.e.v. as $m_{\widetilde{\nu}_{\tau}}^{2} \xi_{\tau} / \sqrt{2}$.

Note that since the decay into the Higgs is strongly suppressed by the phase space factor $\beta_{h}$, and since we will consider in the following mostly a gravitino mass of $150 \mathrm{GeV}$, while $m_{h}>114 \mathrm{GeV}$ for a Standard Model-like Higgs, this channel is negligible in our case, as can be seen from figure 1. We will therefore ignore it in the following discussion, although it will be included in the numerical results and in the figures.

\section{$3 \quad$ Neutrino energy spectrum}

The injection spectrum of neutrinos from gravitino decay is composed of a series of contributions. Firstly, the two-body gravitino decay into a photon and a tau neutrino produces a monoenergetic line at half the gravitino mass:

$$
\frac{d N_{\nu_{\tau}}}{d E}\left(\psi_{3 / 2} \rightarrow \gamma \nu_{\tau}\right) \simeq \delta\left(E-\frac{m_{3 / 2}}{2}\right)
$$

Additionally, the decay into $Z^{0} \nu_{\tau}$ produces a second line at an energy

$$
E_{\nu_{\tau} Z}=\frac{m_{3 / 2}}{2}\left(1-\frac{M_{Z}^{2}}{m_{3 / 2}^{2}}\right)
$$




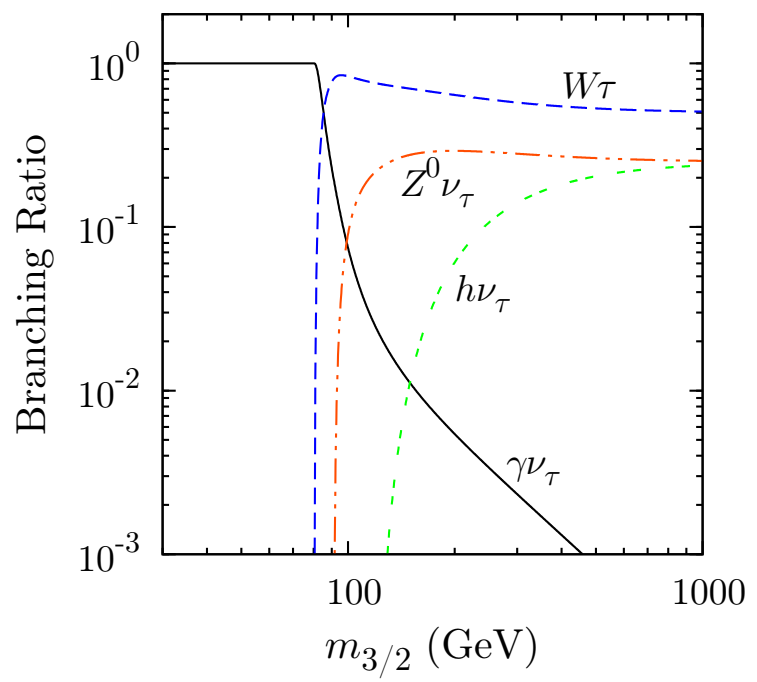

Figure 1: Branching ratios of the different gravitino decay channels as a function of the gravitino mass for the case of large $\mu$ parameter (here taken as $\mu=10 \mathrm{TeV}$ ) and decoupling of the heavy Higgses. The light Higgs mass is taken to be $115 \mathrm{GeV}$. The gaugino mass parameters are taken so as to satisfy the unification relation and to give $M_{1}=1.5 \mathrm{~m}_{3 / 2}$ at the electroweak scale. The sneutrino mass parameter is fixed at $2 m_{3 / 2}$ and $\tan \beta=10$. We see that above the $Z^{0}$ mass threshold, the dominant line from $Z^{0} \nu_{\tau}$ always has a branching ratio larger than $25 \%$.

which is not completely monoenergetic due to the width of the $Z^{0}$ boson. Instead, it is described by a normalised Breit-Wigner profile:

$$
\frac{d N_{\nu_{\tau} Z}}{d E}=\frac{1}{\left(E^{2}-E_{\nu_{\tau} Z}^{2}\right)^{2}+E_{\nu_{\tau} Z}^{2} \Gamma_{\nu_{\tau} Z}^{2}}\left(\int_{0}^{\infty} \frac{d E}{\left(E^{2}-E_{\nu_{\tau} Z}^{2}\right)^{2}+E_{\nu_{\tau} Z}^{2} \Gamma_{\nu_{\tau} Z}^{2}}\right)^{-1},
$$

where

$$
\Gamma_{\nu_{\tau} Z}=\left|\frac{\partial E_{\nu_{\tau} Z}}{\partial M_{Z}}\right| \Gamma_{Z}=\frac{M_{Z}}{m_{3 / 2}} \Gamma_{Z}
$$

Furthermore, the decay into $h \nu_{\tau}$ produces a similar line, with a differential energy spectrum denoted by $d N_{\nu_{\tau} h} / d E$. This line is less prominent due to the suppressed branching ratio into the Higgs decay channel. Lastly, the fragmentation of the massive gauge bosons produces a continuous spectrum of neutrinos in all flavours. We have simulated the fragmentation of the gauge bosons with the event generator PYTHIA 6.4 [19] and extracted the spectra in the different neutrino flavours for the $W^{ \pm}, Z^{0}$ and $h$ channels, which we denote by $d N_{\nu_{x}}^{W} / d E, d N_{\nu_{x}}^{Z} / d E$ and $d N_{\nu_{x}}^{h} / d E$, respectively. The leptonic decays $W \rightarrow l \nu, Z^{0} \rightarrow \nu \nu$ and $h \rightarrow l \nu$ also produce, in the rest frame of the decaying particle, monoenergetic neutrinos in all flavours. However, due to the boost of the gauge bosons in different directions, the lines smear out almost completely in the Earth's rest frame, giving just an additional contribution to the continuous part of the spectrum. Taking the various decay channels into account, the 


\begin{tabular}{|rcccc|}
\hline$m_{3 / 2}$ & $\operatorname{BR}\left(\gamma \nu_{\tau}\right)$ & $\mathrm{BR}\left(W^{ \pm} \tau^{\mp}\right)$ & $\operatorname{BR}\left(Z^{0} \nu_{\tau}\right)$ & $\operatorname{BR}\left(h \nu_{\tau}\right)$ \\
\hline $10 \mathrm{GeV}$ & 1 & - & - & - \\
$85 \mathrm{GeV}$ & 0.53 & 0.47 & - & - \\
$100 \mathrm{GeV}$ & 0.08 & 0.83 & 0.09 & - \\
$150 \mathrm{GeV}$ & 0.01 & 0.70 & 0.28 & 0.01 \\
$250 \mathrm{GeV}$ & 0.003 & 0.60 & 0.29 & 0.11 \\
$1000 \mathrm{GeV}$ & 0.0002 & 0.51 & 0.25 & 0.24 \\
\hline
\end{tabular}

Table 1: Branching ratios into the different gravitino decay channels for a number of specific gravitino masses. The various parameters are chosen as in figure 1.

total spectra for the different neutrino flavours are given by:

$$
\begin{aligned}
\frac{d N_{\nu_{e}}}{d E}= & \operatorname{BR}\left(W^{ \pm} \tau^{\mp}\right) \frac{d N_{\nu_{e}}^{W}}{d E}+\operatorname{BR}\left(Z^{0} \nu_{\tau}\right) \frac{d N_{\nu_{e}}^{Z}}{d E}+\operatorname{BR}\left(h \nu_{\tau}\right) \frac{d N_{\nu_{e}}^{h}}{d E} \\
\frac{d N_{\nu_{\mu}}}{d E}= & \operatorname{BR}\left(W^{ \pm} \tau^{\mp}\right) \frac{d N_{\nu_{\mu}}^{W}}{d E}+\operatorname{BR}\left(Z^{0} \nu_{\tau}\right) \frac{d N_{\nu_{\mu}}^{Z}}{d E}+\operatorname{BR}\left(h \nu_{\tau}\right) \frac{d N_{\nu_{\mu}}^{h}}{d E} \\
\frac{d N_{\nu_{\tau}}}{d E}= & \operatorname{BR}\left(\gamma \nu_{\tau}\right) \delta\left(E-\frac{m_{3 / 2}}{2}\right)+\operatorname{BR}\left(Z^{0} \nu_{\tau}\right) \frac{d N_{\nu_{\tau} Z}}{d E}+\operatorname{BR}\left(h \nu_{\tau}\right) \frac{d N_{\nu_{\tau} h}}{d E} \\
& +\operatorname{BR}\left(W^{ \pm} \tau^{\mp}\right) \frac{d N_{\nu_{\tau}}^{W}}{d E}+\operatorname{BR}\left(Z^{0} \nu_{\tau}\right) \frac{d N_{\nu_{\tau}}^{Z}}{d E}+\operatorname{BR}\left(h \nu_{\tau}\right) \frac{d N_{\nu_{\tau}}^{h}}{d E} .
\end{aligned}
$$

The branching ratios for the different decay channels can be straightforwardly computed from the decay widths, eq. (2.3) and (2.6). They turn out to depend mainly on the gravitino mass, with a milder dependence on the ratio between the gaugino masses $M_{1}$ and $M_{2}$ at the electroweak scale. For illustration, we show in figure 1 the branching ratios as a function of the gravitino mass for the case of a large $\mu$ parameter, unified gaugino masses with $M_{1}=1.5 m_{3 / 2}$ and $m_{\widetilde{\nu}}=2 m_{3 / 2}$ at the electroweak scale, and $\tan \beta=10$. In addition, we list in table 1 , the branching ratios into the different decay channels for a number of specific gravitino masses. Note also that in all of the parameter space above the $Z^{0}$ threshold, at least two neutrino lines are present with more than $1 \%$ branching ratio. Actually, sufficiently above the Higgs threshold, the neutrino lines from the Higgs and $Z^{0}$ decay channels have comparable strength.

The energy spectra for the different neutrino flavours, which were obtained as described above, are shown in figure 2. In this case, a gravitino mass of $m_{3 / 2}=150 \mathrm{GeV}$ was used, and for that value, all three lines mentioned above are visible in the spectrum. We see that the tau neutrino spectrum shows a very characteristic signature of two or three distinctive peaks (three in the region above the lightest Higgs threshold, as in this case) in addition to a continuum which is suppressed at low energies. The spectra of the other two flavours are very similar to each other, consisting only of a continuum contribution practically following a power law behaviour $\propto E^{-2}$ below the sharp threshold at half the gravitino mass.

\section{Neutrino fluxes}

In this section we will consider the diffuse neutrino background at the Earth arising from the neutrino spectrum from gravitino dark matter decay as discussed above. The diffuse neutrino flux has two sources: the decay of gravitinos at cosmological distances and the decay of gravitinos in the Milky Way halo. The former contribution is perfectly isotropic, while the latter has a mild dependence on the Galactic coordinates $[10,11]$. 


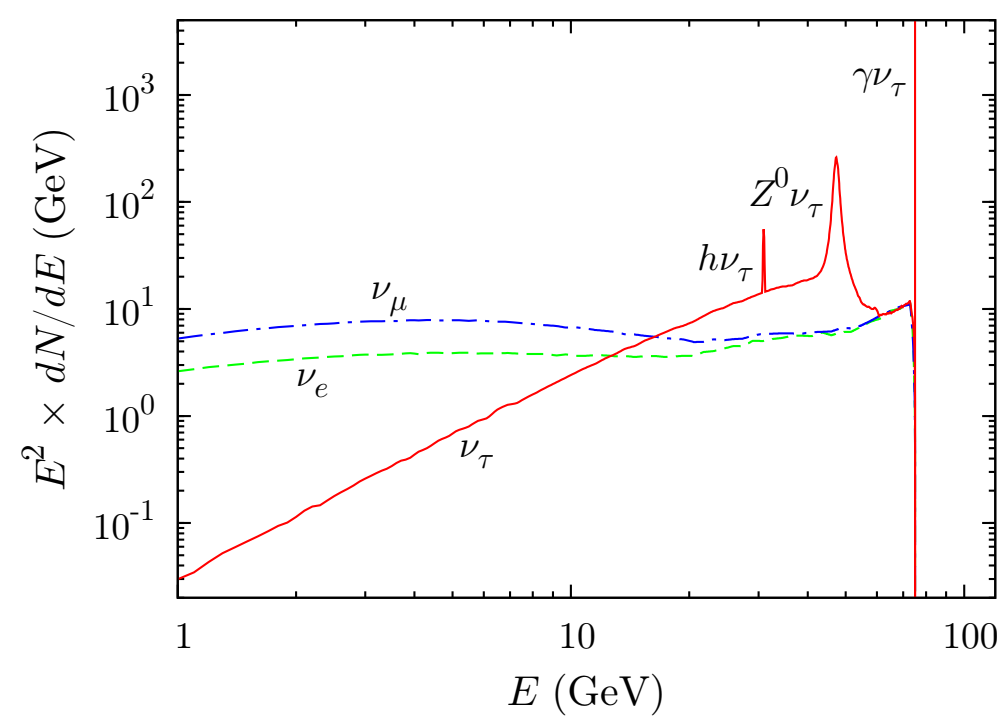

Figure 2: Neutrino injection energy spectra from gravitino decay for the different flavours in the case of $m_{3 / 2}=150 \mathrm{GeV}$. The $Z^{0}$ line is clearly visible at an energy of $47 \mathrm{GeV}$. We consider here a Higgs mass of $115 \mathrm{GeV}$, giving a peak at $\sim 30 \mathrm{GeV}$.

The decay of gravitinos at a comoving distance $\chi(z)$, where $z$ denotes redshift, produces a neutrino flux with a redshifted energy spectrum $d N_{\nu} / d(y E)$, with $y=1+z$. Making use of the variation of comoving distance with respect to redshift in a matter- and dark energy-dominated Universe, $d \chi / d z=(1+z)^{-3 / 2} /\left(a_{0} H_{0} \sqrt{\Omega_{M}\left(1+\kappa(1+z)^{-3}\right)}\right)$, with $a_{0}$ and $H_{0}$ being the present cosmic scale factor and the Hubble parameter, respectively, and $\kappa=\Omega_{\Lambda} / \Omega_{M} \simeq 3$ being the ratio between the vacuum and matter density parameters, it is straightforward to show that the neutrino flux received at the Earth reads:

$$
\frac{d J_{\mathrm{eg}}}{d E} \simeq A_{\mathrm{eg}} \int_{1}^{\infty} d y \frac{d N_{\nu}}{d(y E)} \frac{y^{-3 / 2}}{\sqrt{1+\kappa y^{-3}}},
$$

where most neutrinos come from very low redshifts. In this equation,

$$
A_{\mathrm{eg}}=\frac{\Omega_{3 / 2} \rho_{c}}{4 \pi \tau_{3 / 2} m_{3 / 2} H_{0} \Omega_{M}^{1 / 2}}=1.1 \times 10^{-7}\left(\mathrm{~cm}^{2} \mathrm{~s} \mathrm{sr}\right)^{-1}\left(\frac{\tau_{3 / 2}}{1.3 \times 10^{26} \mathrm{~s}}\right)^{-1}\left(\frac{m_{3 / 2}}{150 \mathrm{GeV}}\right)^{-1}
$$

where we have taken the gravitino density to be equal to the cold dark matter density, $\Omega_{3 / 2} h^{2}=0.1$, and the other constants are the critical density $\rho_{c}=1.05 h^{2} \times 10^{-5} \mathrm{GeV} \mathrm{cm}^{-3}$, the matter density parameter $\Omega_{M}=0.25$ and $H_{0}=100 h \mathrm{~km} \mathrm{~s}^{-1} \mathrm{Mpc}^{-1}$ with $h=0.73$.

In addition to the extragalactic signal there exists a slightly anisotropic neutrino flux stemming from the decay of gravitinos in the Milky Way halo. The energy spectrum is given by

$$
\frac{d J_{\text {halo }}}{d E}=A_{\text {halo }} \frac{d N_{\nu}}{d E},
$$

where the intensity of the flux, $A_{\text {halo }}$, depends on the direction of observation. It is proportional to the line-of-sight integration over the halo density profile $\rho_{\text {halo }}$, being defined as

$$
A_{\text {halo }}=\frac{1}{4 \pi \tau_{3 / 2} m_{3 / 2}} \int_{\text {l.o.s. }} \rho_{\text {halo }}(\vec{l}) d \vec{l} \text {. }
$$


For our numerical analysis, we will adopt the spherically symmetric Navarro, Frenk and White (NFW) profile [20]:

$$
\rho_{\text {halo }}(r)=\frac{\rho_{0}}{\left(r / r_{c}\right)\left[1+\left(r / r_{c}\right)\right]^{2}},
$$

with $\rho_{0}=0.26 \mathrm{GeV} / \mathrm{cm}^{3}$ and $r_{c}=20 \mathrm{kpc}$. The normalisation is chosen such that $\rho\left(r_{\odot}\right)=$ $0.3 \mathrm{GeV} / \mathrm{cm}^{3}$, where $r_{\odot}=8.5 \mathrm{kpc}$ is the distance of the Sun from the Galactic centre. Our conclusions will turn out to be rather insensitive to the particular choice of the halo profile due to the linear dependence of the neutrino fluxes on the dark matter density along the line-of-sight and the fact that we will integrate the signal over the whole sky excluding the Galactic disk.

After being produced in gravitino decays, neutrinos propagate while undergoing flavour oscillations. Since neutrinos typically travel very long distances before reaching us, the conversion probabilities are [21]:

$$
\begin{aligned}
P\left(\nu_{e} \leftrightarrow \nu_{\mu}\right) & =\frac{1}{2}\left(s_{23}^{2} \sin ^{2} 2 \theta_{13}+c_{23}^{2} \sin ^{2} 2 \theta_{12}\right), \\
P\left(\nu_{e} \leftrightarrow \nu_{\tau}\right) & =\frac{1}{2}\left(c_{23}^{2} \sin ^{2} 2 \theta_{13}+s_{23}^{2} \sin ^{2} 2 \theta_{12}\right), \\
P\left(\nu_{\mu} \leftrightarrow \nu_{\tau}\right) & =\frac{1}{2}\left(c_{13}^{4} \sin ^{2} 2 \theta_{23}-s_{23}^{2} c_{23}^{2} \sin ^{2} 2 \theta_{12}\right),
\end{aligned}
$$

while the survival probabilities are

$$
\begin{aligned}
& P\left(\nu_{e} \leftrightarrow \nu_{e}\right)=1-\frac{1}{2}\left(\sin ^{2} 2 \theta_{13}+c_{13}^{4} \sin ^{2} 2 \theta_{12}\right), \\
& P\left(\nu_{\mu} \leftrightarrow \nu_{\mu}\right)=1-\frac{1}{2}\left(4 c_{13}^{2} s_{23}^{2}\left(1-c_{13}^{2} s_{23}^{2}\right)+c_{23}^{4} \sin ^{2} 2 \theta_{12}\right), \\
& P\left(\nu_{\tau} \leftrightarrow \nu_{\tau}\right)=1-\frac{1}{2}\left(4 c_{13}^{2} c_{23}^{2}\left(1-c_{13}^{2} c_{23}^{2}\right)+s_{23}^{4} \sin ^{2} 2 \theta_{12}\right),
\end{aligned}
$$

with $s_{i j} \equiv \sin \theta_{i j}$ and $c_{i j} \equiv \cos \theta_{i j}$. Inserting into these equations the experimental best fit values for the neutrino mixing angles $\sin ^{2} \theta_{12}=0.304, \sin ^{2} \theta_{23}=0.5$ and $\sin ^{2} \theta_{13}=0.01$ [22], we finally obtain

$$
\begin{aligned}
P\left(\nu_{e} \leftrightarrow \nu_{e}\right) & =0.56, \\
P\left(\nu_{e} \leftrightarrow \nu_{\mu}\right)=P\left(\nu_{e} \leftrightarrow \nu_{\tau}\right) & =0.22, \\
P\left(\nu_{\mu} \leftrightarrow \nu_{\mu}\right)=P\left(\nu_{\mu} \leftrightarrow \nu_{\tau}\right)=P\left(\nu_{\tau} \leftrightarrow \nu_{\tau}\right) & =0.39 .
\end{aligned}
$$

Thus, even when the primary neutrino flux is originally mainly composed of tau neutrinos, the flavour oscillations during the propagation will produce comparable fluxes of electron, muon and tau neutrinos due to the large neutrino mixing angles. In particular, due to the maximal atmospheric mixing angle, the fluxes of muon and tau neutrinos are expected to be essentially identical.

The fluxes for the different neutrino flavours and their extragalactic and halo contributions are shown in figure 3. In this plot, a band of $\pm 10^{\circ}$ around the Galactic disk has been removed, and the spectrum is shown with a $10 \%$ energy resolution in order to take the finite energy resolution of the detector into account. Note that even with this optimistic assumption for the energy resolution, the lines from the decay into $\gamma \nu_{\tau}$ and $h \nu_{\tau}$ become practically indistinguishable from the continuum, whereas the line from the decay into $Z^{0} \nu_{\tau}$ can be resolved. Its position could allow a determination of the gravitino mass, even without determining the endpoint of the spectrum. 


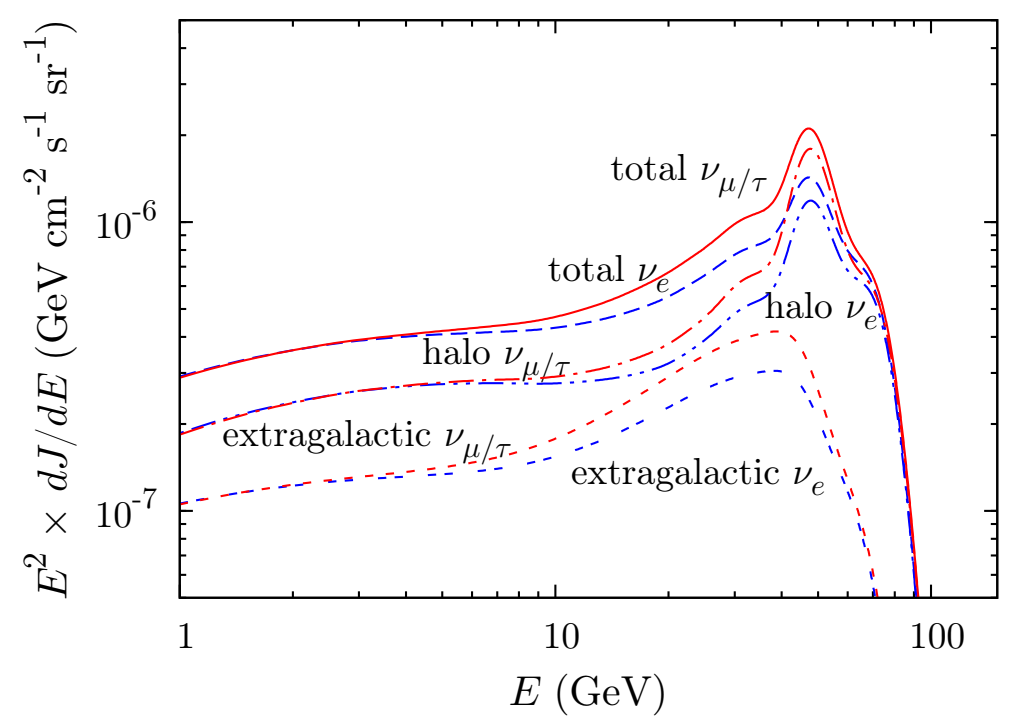

Figure 3: Total neutrino fluxes, as well as the extragalactic and halo contributions for the different neutrino flavours after propagation using an energy resolution of $10 \%$. The gravitino mass and lifetime are chosen to be $m_{3 / 2}=150 \mathrm{GeV}$ and $\tau_{3 / 2} \simeq 10^{26} \mathrm{~s}$.

\section{$5 \quad$ Neutrino backgrounds}

The detection of a possible neutrino signal from gravitino decay is hindered by considerable neutrino backgrounds. Namely, in the energy range of interest, there exist large background neutrino fluxes produced by interactions of cosmic rays with the Earth's atmosphere or with the solar corona, as well as neutrino fluxes from distant Galactic sources. Let us briefly discuss these backgrounds separately.

The collision of energetic cosmic rays with nuclei in the upper atmosphere produces showers of hadrons, mostly pions, that in turn produce in each decay two muon neutrinos and one electron neutrino. Neutrinos arrive from all directions at the detector site after propagating a distance ranging between $\sim 10$ and $12800 \mathrm{~km}$ while undergoing flavour oscillations. The electron and muon neutrino fluxes have been carefully computed, assuming massless neutrinos, by Battistoni et al. with the Monte Carlo simulation package FLUKA [23]. The theoretical uncertainty of the neutrino flux is estimated to be better than a $20 \%$ over the energy range of interest for this paper [24].

The effects of neutrino oscillations on the flavour composition of these fluxes can easily be included using the following expression for the conversion probability of muon neutrinos into tau neutrinos:

$$
P\left(\nu_{\mu} \rightarrow \nu_{\tau}\right)=\sin ^{2} 2 \theta_{\mathrm{atm}} \sin ^{2}\left(1.27 \frac{\Delta m_{\mathrm{atm}}^{2}\left[\mathrm{eV}^{2}\right] L[\mathrm{~km}]}{E[\mathrm{GeV}]}\right) .
$$

In this expression, $E$ is the neutrino energy and $L$ is their propagation length after being produced in the atmosphere, which is given by

$$
L=\sqrt{\left(R_{\oplus} \cos \theta\right)^{2}+2 R_{\oplus} h+h^{2}}-R_{\oplus} \cos \theta,
$$

with $R_{\oplus}=6371 \mathrm{~km}$ being the mean Earth radius and $h=15 \mathrm{~km}$ the mean altitude at which atmospheric muon neutrinos are produced. Moreover, the neutrino parameters relevant for the atmospheric oscillations are $\sin ^{2} 2 \theta_{\mathrm{atm}}=1,\left|\Delta m_{\mathrm{atm}}^{2}\right|=2.4 \times 10^{-3} \mathrm{eV}^{2}[22]$. 


\begin{tabular}{|cccc|}
\hline Flavour & $N_{0}$ & $\gamma$ & $A$ \\
\hline$\nu_{e}+\bar{\nu}_{e}$ & $7.4 \times 10^{-6}$ & 2.03 & $8.5 \times 10^{-6}$ \\
$\nu_{\mu}+\bar{\nu}_{\mu}$ & $1.3 \times 10^{-5}$ & 1.98 & $8.5 \times 10^{-6}$ \\
\hline
\end{tabular}

Table 2: Values for the parametrisation of the corona electron and muon neutrino flux.

In addition to the flux of tau neutrinos originating from the conversion of muon neutrinos, there exists an intrinsic contribution from the decay of charmed particles produced in the atmosphere, coming from all directions, which has a size about $10^{6}$ times smaller than the flux of electron and muon neutrinos from pion decay. This intrinsic contribution has been computed by Pasquali and Reno [25] and can be parametrised as

$$
\log _{10}\left[E^{3} \frac{d J_{\nu_{\tau}}}{d E} /\left(\frac{\mathrm{GeV}^{2}}{\mathrm{~cm}^{2} \mathrm{ssr}}\right)\right]=-A+B x-C x^{2}-D x^{3},
$$

where $x=\log _{10}(E[\mathrm{GeV}]), A=6.69, B=1.05, C=0.150$ and $D=-0.00820$. The next-toleading order QCD calculation also shown in their paper gives lower fluxes for energies below several $\mathrm{TeV}$ and is therefore less conservative.

Analogous to the production of neutrinos in the Earth's atmosphere, neutrinos are produced in the solar corona by cosmic-ray collisions. This neutrino flux has been studied by Ingelman and Thunman in [26], who found that the flux of electron and muon neutrinos intergrated over the solar disk can be described by the following parametrisation:

$$
\frac{d \phi_{x}}{d E}=N_{0} \frac{(E[\mathrm{GeV}])^{-\gamma-1}}{1+A(E[\mathrm{GeV}])}\left(\mathrm{GeV} \mathrm{cm}^{2} \mathrm{~s}\right)^{-1},
$$

which is valid for $10^{2} \mathrm{GeV} \leq E \leq 10^{6} \mathrm{GeV}$. The numerical values of the coefficients $N_{0}, A$ and $\gamma$ can be found in table 2 for $x=\nu_{e}+\bar{\nu}_{e}, \nu_{\mu}+\bar{\nu}_{\mu}$. The electron and muon neutrinos and antineutrinos produced in the solar corona oscillate during their propagation to the Earth. In view of the long distance travelled, the conversion and survival probabilities can be averaged, and the fluxes at the Earth in the different flavours can be straightforwardly calculated from eq. (4.8).

Lastly, the fluxes of tau neutrinos that originate from Galactic sources are discussed by Athar, Lee and Lin in [27]. For the tau neutrino flux from the Galactic plane in the presence of neutrino oscillations they find the parametrisation

$$
\frac{d J_{\nu_{\tau}}}{d E}=9 \times 10^{-6}\left(\mathrm{GeV} \mathrm{cm}^{2} \mathrm{~s} \mathrm{sr}\right)^{-1}(E[\mathrm{GeV}])^{-2.64},
$$

which is valid in the energy range $1 \mathrm{GeV} \leq E \leq 10^{3} \mathrm{GeV}$.

\section{Detection prospects}

\subsection{Comparison with the signal}

The full-sky signal for the neutrinos from gravitino decay is shown in figure 4 together with the results for the atmospheric background from FLUKA. The signal lies several orders of magnitude below the expected atmospheric background for all flavours. Therefore, we find that the interpretation of the EGRET and HEAT anomalies in terms of gravitino decay is compatible with neutrino flux measurements, as it does not lead to an overproduction of neutrinos. 


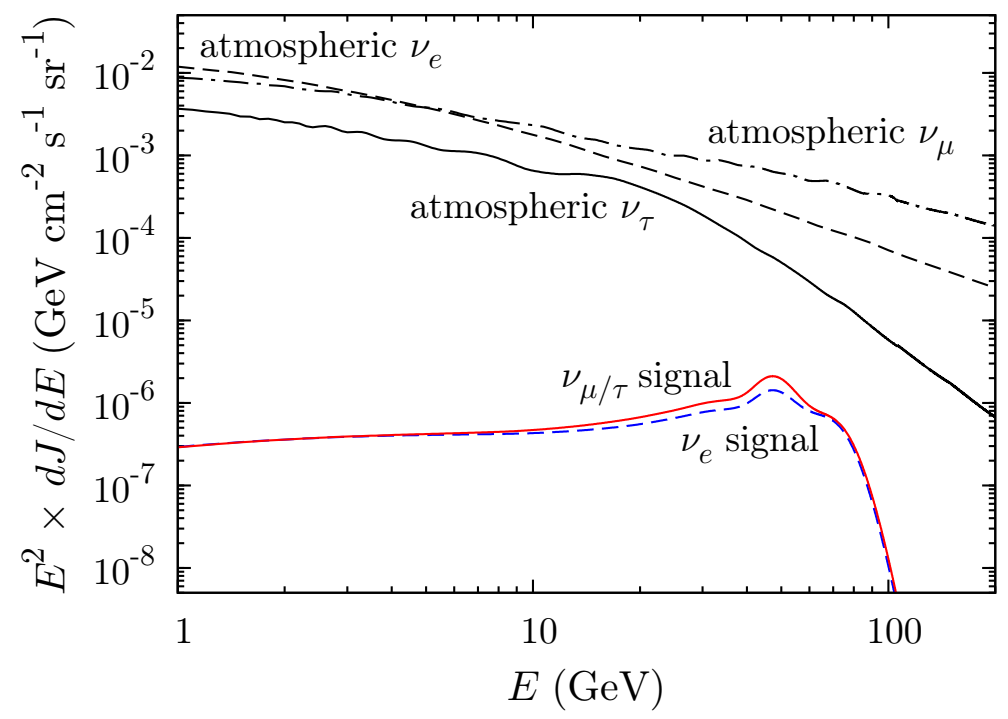

Figure 4: Full-sky neutrino fluxes expected at the Super-K site for the same gravitino mass and lifetime as in figure 3. The energy resolution is taken to be $10 \%$, which is insufficient to resolve the different peaks in the spectrum.

Going beyond this consistency check, we examine in the following the possibility of detecting this exotic contribution in neutrino experiments. Due to the low signal-to-background ratio, the signal cannot be detected directly. It will therefore be necessary to find strategies for effectively reducing the background in order to have any chance of detecting the signal. As is apparent from figure 4, the tau neutrino channel appears to be the most promising of the three flavours, since it has the lowest background. In general, the neutrino spectrum from gravitino decay has some very specific features that could allow to distinguish it from the featureless backgrounds, but the question is whether neutrino detectors will be able to reach sufficient sensitivity to resolve these features.

\subsection{Electron and muon neutrinos}

For the electron and muon neutrinos, which are more easily detected in neutrino observatories, the signal-to-background ratio is very low $\left(\sim 10^{-3-4}\right)$ for lifetimes that are not already excluded by gamma rays [10,11] or antimatter detection $[12,13]$. As can be seen from figure 4 , even the peak of the spectrum is three orders of magnitude below the background. This makes distinguishing an exotic signal from the background extremely difficult.

Unfortunately, we could not find a suitable strategy to sufficiently reduce this background, e.g. by exploiting directionality. In general the atmospheric neutrino baseline is too short for all muon neutrinos to oscillate into another flavour at energies of order $50-100 \mathrm{GeV}$. It therefore seems hopeless to try to detect the signal without having prior knowledge of the position of the peak in the gravitino decay spectrum. In case information on the line is available, e.g. from the detection of a monochromatic gamma-ray line by the Fermi GammaRay Space Telescope (FGST, formerly named GLAST) [28], then one could perhaps envisage strategies to disentangle the signal from the background. However, that would probably require a much better knowledge of the atmospheric neutrino flux at the relevant energies and a better energy resolution than is presently available. 

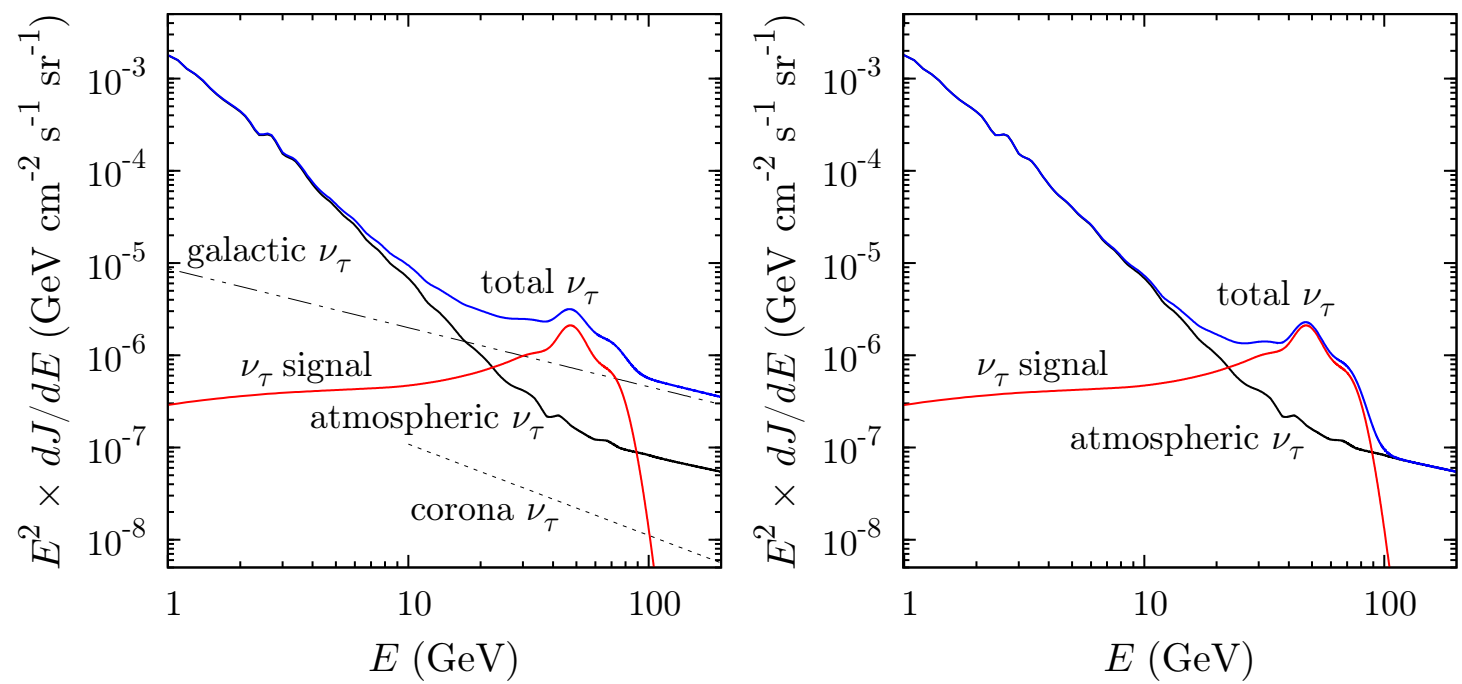

Figure 5: Down-going tau neutrino fluxes expected at the Super-K site with (left) and without (right) the contribution from the Galactic disk and the solar corona for the same gravitino mass and lifetime as in figure 3 .

\subsection{Tau neutrinos}

For the tau neutrinos, the signal-to-background ratio is more promising, since it lies above $\sim$ $10^{-2}$ at the peak energy. Moreover, most of the background of tau neutrinos from atmospheric oscillations and also the other two subdominant sources of tau neutrinos can be effectively reduced by exploiting directionality. In fact, the solar corona neutrinos and the Galactic neutrinos mostly come either from the direction of the Sun or from the Galactic plane, which could be excluded from the search to reduce the backgrounds. Furthermore, in our energy range the tau neutrinos arising from oscillations of the original muon atmospheric neutrinos are mostly generated for oscillation lengths of the order of the Earth diameter. This means that we expect a very low tau neutrino background if we only consider the flux arriving at the detector from above the horizon. In this way, the background of tau neutrinos can be reduced by several orders of magnitude. In figure 5, we show the fluxes for down-going tau neutrinos at the Super-Kamiokande site. We see that in this case, the signal can exceed the simulated background from FLUKA, even without cutting away the Sun or the Galactic plane.

\subsection{Observability in Super-Kamiokande}

In Cherenkov telescopes, tau neutrinos can only be observed via charged current (CC) interactions and are very difficult to disentangle from the other flavours, since the Cherenkov signal is not sufficiently distinctive to allow identification on an event-by-event basis.

The Super-Kamiokande collaboration has developed a statistical method to discriminate tau neutrinos from the background of other flavours [29]. Using two different strategies, namely a likelihood analysis and a neural network, they find an efficiency of $43.1 \%$ and $39.0 \%$, respectively, to identify tau neutrinos correctly. However, they still misidentify $3.8 \%$ and $3.4 \%$, respectively, of the electron and muon background neutrinos as tau neutrinos. Due to the large number of electron and muon neutrino events, the sample of tau neutrinos is dominated by misidentified neutrinos. The true tau neutrino events can therefore only be extracted on a statistical basis using Monte Carlo methods. In the end, the data is found to 


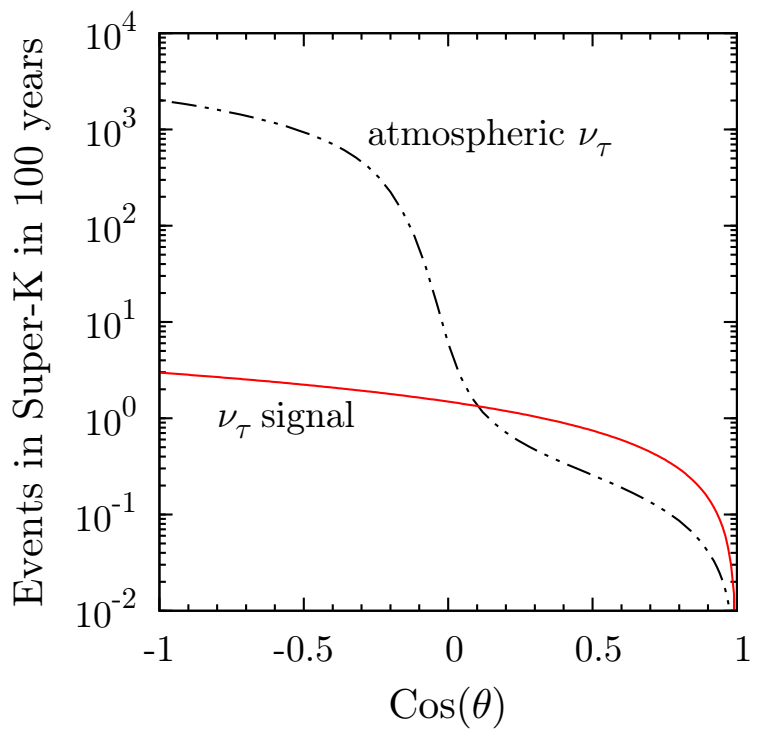

Figure 6: Events in 100 years of observation in Super-K due to CC interactions of $\nu_{\tau}$ and $\bar{\nu}_{\tau}$ from the atmosphere (dash-dotted) and from gravitino decay (solid), integrating the signal from the zenith direction to the angle $\theta$. The gravitino mass and lifetime are chosen as in figure 3 .

be consistent with the atmospheric tau neutrino flux and neutrino oscillations: The full-sky atmospheric tau neutrino signal results in fact in an expected 78 events in the Super-K I period and 43 events in the Super-K II period [29, 30].

However, this analysis does not exploit the information about the spectral shape of the signal apart from setting a threshold for $\tau$ lepton production, i.e. $E_{\nu_{\tau}}>m_{\tau}+m_{\tau}^{2} / 2 m_{n} \simeq$ $3.5 \mathrm{GeV}$, so this kind of data analysis could certainly be improved to search for a signal with a peak above the continuum, as in our case.

Despite the experimental difficulties, it is worthwhile to examine the theoretically expected signal in the tau channel. Figure 6 shows the expected number of tau neutrino and antineutrino events per century of observation at Super-Kamiokande within a zenith angle integrated from $\cos \theta$ to 1 . If only down-going neutrinos are selected, the signal from gravitino decays lies above the atmospheric background for higher energies. However, the fluxes are extremely low and result in only a few events per century, making it practically impossible to discriminate them from the other flavours using statistical methods.

One detector specifically optimised for measuring tau neutrinos above $17 \mathrm{GeV}$ event-byevent is OPERA [31], which is already active in Gran Sasso and will measure tau neutrino appearance in a muon neutrino beam produced at CERN. Unfortunately, the detector's effective mass is more than a factor 10 smaller than that of Super-K and thus, even neglecting the issue of directionality, would be able to observe only one event from gravitino decays in more than 1000 years.

We therefore conclude that present detectors are unable to detect the signal, either because they do not have a sufficiently good efficiency for identifying tau neutrinos, or because they are too small for the low intensity of our signal, or both. 


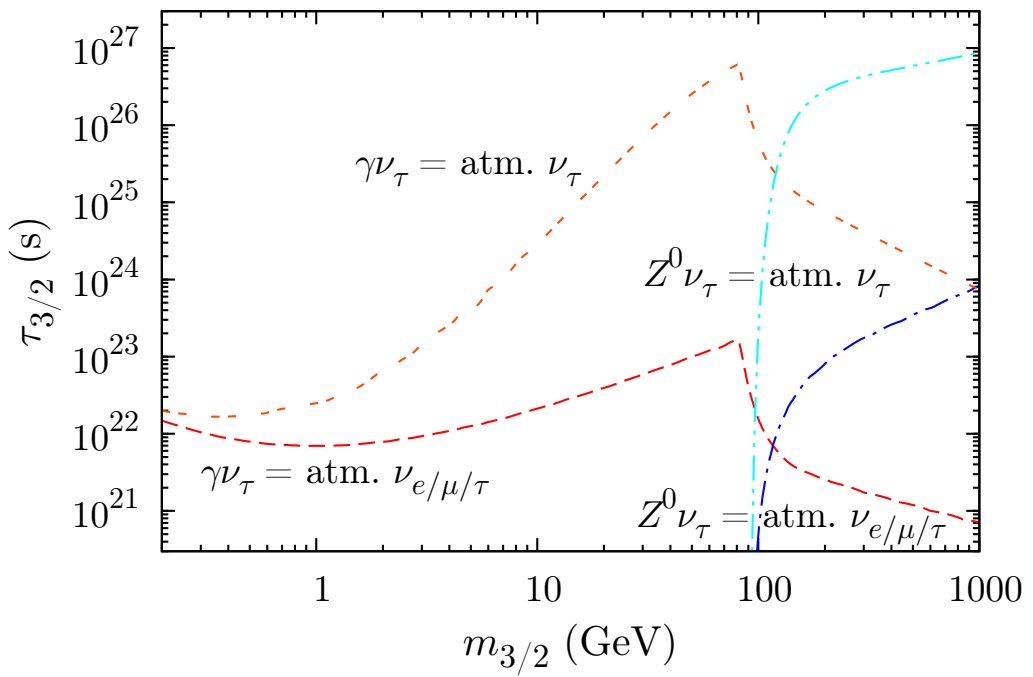

Figure 7: Region of the gravitino lifetime where the line signals from the two-body decays into $\gamma \nu_{\tau}$ and $Z^{0} \nu_{\tau}$ overcome the atmospheric background either for all neutrino flavours or for down-going tau neutrinos only. We see that considering the tau flavour and exploiting directionality can improve the detectability by three orders of magnitude for large gravitino masses. We use here the branching ratios shown in figure 1 for the two lines. Note that in the intermediate region the continuum spectrum from $W^{ \pm}$fragmentation may be used to close the gap, but we do not consider this possibility here.

\section{$7 \quad$ Future detectors}

In this section, we will briefly discuss the prospect of detection for the future. In order to consider the general parameter space for gravitino decay, we show in figure 7 the region of gravitino lifetime and mass where the neutrino signal in the $Z^{0}$ and gamma peaks is equal to the simulated atmospheric background. For the muon/electron neutrino flux, this result is similar to that presented in [32], except that we are weighting the channels with the gravitino branching ratios and that we have only one neutrino produced in the line instead of two. Real limits from neutrino experiments have been considered in the past in [33].

We are taking here as in [32] a nominal energy resolution of 0.3 in $\log _{10}(E[\mathrm{GeV}])$ around the peak position and we single out the value of the lifetime for which the peak is equal to the background. Note that since the signal is proportional to $1 / \tau_{3 / 2}$, requiring the peak to be larger than the background by a specific factor only rescales the curves by the inverse of this factor.

We clearly see again that the tau neutrino channel in the down-going direction allows to constrain the gravitino lifetime a few orders of magnitude better than the muon or electron neutrino fluxes. On the other hand, similar plots for the gamma-ray channel [10] are even more sensitive and give bounds at the order of $10^{27} \mathrm{~s}$ for gravitino masses below the $W^{ \pm}$and $Z^{0}$ thresholds. For masses above $200 \mathrm{GeV}$, the tau neutrino channel starts to compete in sensitivity with the photon channel, if we neglect for the moment the difficulties connected with measuring such a low flux and identifying the neutrino flavour. At even higher energies the background flux decreases quickly and therefore the signal-to-background ratio improves, but the signal rate then also decreases (as $1 / m_{3 / 2}$ ), making detection more difficult. 


\subsection{Hyper-Kamiokande}

The prospects for Hyper-Kamiokande can be easily obtained by considering that its mass is planned to be a factor of 10 (for the 0.5 megaton project) to 20 (for a 1 megaton case) larger than Super-Kamiokande. Assuming that the rest of the detector performances are unchanged, we expect to find approximately 20-40 events from our signal per century from the upper hemisphere. ${ }^{1}$

This number of events might still be too small to allow for statistical analysis. However, we expect most of the events to appear within the peak region or near the threshold and therefore, an appropriate energy binning, especially optimised after a signal has already been detected in gamma rays, could allow to collect a significant number of events above the background in a specific energy bin on a shorter timescale. Still, it is clear that a sufficiently good energy resolution is a key requirement for singling out the line events, and it remains uncertain how and if the tau statistical discrimination analysis can be applied to a sample of such few events.

\subsection{IceCube and $\mathrm{km}^{3}$ detectors}

Detectors of $\mathrm{km}^{3}$ dimensions have in principle sufficient size to collect enough events to detect the signal within a reasonable time span. Even considering that IceCube is actually looking downwards and not at the upper hemisphere, from the horizontal direction and the proton cross-section we estimate $\mathcal{O}(100)$ events per year for the completed experiment. Of course, the effective area depends on the neutrino energy: taking the effective area given in [34] for the opposite direction and assuming most of the signal is above $100 \mathrm{GeV}$, we have instead $\mathcal{O}(10)$ events per year. In general, it would be desirable to lower the energy threshold to reach below $100 \mathrm{GeV}$ in order to cover the energy range favoured by the EGRET and HEAT anomalies. The combination of IceCube with AMANDA already allows to lower the threshold to $30 \mathrm{GeV}$. Additionally, plans are being considered for adding another, denser subdetector at a deeper location to improve the sensitivity to dark matter annihilations [34]. Such a configuration could probably also be useful for investigating the present scenario and, more generally, other decaying dark matter candidates.

However, in the case of Cherenkov detectors, the discrimination of tau neutrinos from other neutrino flavours is generally difficult, and for IceCube strategies for tau flavour identification have been proposed only for neutrinos well above $\mathrm{TeV}$ energies [35]. It could therefore be more favourable to improve the energy resolution and exploit the muon neutrino final state instead.

\section{Conclusions}

We have examined the neutrino spectrum from the decay of unstable gravitino dark matter in a scenario with bilinear $R$-parity violation. It has been pointed out in the recent literature that the decay of gravitino dark matter particles with a lifetime of $\sim 10^{26} \mathrm{~s}$ and a mass of $\sim 150 \mathrm{GeV}$ into massive gauge bosons may account for the anomalies observed in the diffuse extragalactic gamma-ray spectrum as measured by EGRET as well as in the positron fraction as measured by HEAT. ${ }^{2}$ Motivated by this observation, we have computed the neutrino

\footnotetext{
${ }^{1}$ This number could be larger if the fiducial volume of Hyper-Kamiokande is larger than $\sim 1 / 2$ the total volume as it is in Super-Kamiokande.

${ }^{2}$ The existence of a positron excess seems to be supported by preliminary results from PAMELA [36].
} 
spectrum for the same choice of parameters as a consistency check of this scenario. We find that this spectrum is compatible with results from neutrino experiments.

We have also examined the detectability of this exotic component of the neutrino flux to find an independent way to test this scenario. While the signal in the neutrino spectrum with two or more distinct peaks, resulting from two-body gravitino decays into gauge/Higgs boson and neutrino, is very characteristic, it will be challenging to detect these features in neutrino experiments. On one side, present neutrino detectors do not achieve a sufficiently high energy resolution to resolve the subdominant peaks, and on the other side, the event rate is expected to be so small that the background of atmospheric neutrinos overwhelms the signal in all flavours. The most promising signal-to-background ratio is found in the tau neutrino flavour, especially when analysing only the flux from the upper hemisphere since there the atmospheric tau neutrino flux is vastly reduced. However, tau neutrinos are difficult to identify in Cherenkov detectors and probably only an event-by-event identification procedure could allow the signal to be seen with such extremely limited statistics. At present, therefore, it is not possible to detect this contribution due to technological limitations.

The ideal detector for testing the present scenario would be one of megaton mass with the ability to identify and measure tau neutrinos event by event. Should such a detector ever become available, it could be worthwhile to look for this component of the neutrino flux by employing strategies for background reduction such as the ones discussed here, especially if the anomalous signatures in the positron fraction and the diffuse extragalactic gamma-ray spectrum are confirmed by PAMELA and FGST, respectively. The detection of a signal in neutrinos compatible with signals in the other indirect detection channels would in fact bring significant support to the scenario of decaying dark matter, possibly consisting of gravitinos that are unstable due to bilinear $R$-parity violation.

\section{Acknowledgments}

We would like to thank Wilfried Buchmüller, Marco Cirelli, Concha González-García, Ricard Tomàs and Mark Vagins for useful discussions.

LC would like to thank NORDITA and the organisers of the NORDITA program on "TeV scale physics and dark matter" for hospitality and support during part of this work. LC also acknowledges the support of the "Impuls- und Vernetzungsfond" of the Helmholtz Association under the contract number VH-NG-006 and of the European Network of Theoretical Astroparticle Physics ILIAS/N6 under contract number RII3-CT-2004-506222. The work of AI and DT was partially supported by the DFG cluster of excellence Origin and Structure of the Universe.

\section{References}

[1] G. Bertone, D. Hooper and J. Silk, Particle dark matter: evidence, candidates and constraints, Phys. Rept. 405 (2005) 279 [hep-ph/0404175] [SPIRES].

[2] H. Pagels and J.R. Primack, Supersymmetry, cosmology and new TeV physics, Phys. Rev. Lett. 48 (1982) 223 [SPIRES].

[3] J.R. Ellis, D.V. Nanopoulos and S. Sarkar, The cosmology of decaying gravitinos, Nucl. Phys. B 259 (1985) 175 [SPIRES]; 
D. Lindley, Cosmological constraints on the lifetime of massive particles, Astrophys. J. 294 (1985) 1 [SPIRES];

M.H. Reno and D. Seckel, Primordial nucleosynthesis: the effects of injecting hadrons, Phys. Rev. D 37 (1988) 3441 [SPIRES];

S. Dimopoulos, R. Esmailzadeh, L.J. Hall and G.D. Starkman, Is the universe closed by baryons? Nucleosynthesis with a late decaying massive particle,

Astrophys. J. 330 (1988) 545 [SPIRES];

R.J. Scherrer and M.S. Turner, Primordial nucleosynthesis with decaying particles. 1. Entropy producing decays. 2. Inert decays, Astrophys. J. 331 (1988) 19 Astrophys. J. 331 (1988) 33 [SPIRES];

J.R. Ellis, G.B. Gelmini, J.L. Lopez, D.V. Nanopoulos and S. Sarkar, Astrophysical constraints on massive unstable neutral relic particles, Nucl. Phys. B 373 (1992) 399 [SPIRES];

For a review see S. Sarkar, Big bang nucleosynthesis and physics beyond the standard model, Rept. Prog. Phys. 59 (1996) 1493 [hep-ph/9602260] [SPIRES].

[4] M. Pospelov, Particle physics catalysis of thermal big bang nucleosynthesis, Phys. Rev. Lett. 98 (2007) 231301 [hep-ph/0605215] [SPIRES];

R.H. Cyburt, J.R. Ellis, B.D. Fields, K.A. Olive and V.C. Spanos, Bound-state effects on light-element abundances in gravitino dark matter scenarios, JCAP 11 (2006) 014 [astro-ph/0608562] [SPIRES];

M. Kawasaki, K. Kohri, T. Moroi and A. Yotsuyanagi, Big-bang nucleosynthesis and gravitino, Phys. Rev. D 78 (2008) 065011 [arXiv:0804.3745] [SPIRES];

M. Pospelov, J. Pradler and F.D. Steffen, Constraints on supersymmetric models from catalytic primordial nucleosynthesis of beryllium, JCAP 0811 (2008) 020 [arXiv:0807.4287] [SPIRES].

[5] K. Hamaguchi, T. Hatsuda, M. Kamimura, Y. Kino and T.T. Yanagida, Stau-catalyzed ${ }^{6}$ Li production in big-bang nucleosynthesis, Phys. Lett. B 650 (2007) 268 [hep-ph/0702274] [SPIRES].

[6] M. Bolz, A. Brandenburg and W. Buchmüller, Thermal production of gravitinos, Nucl. Phys. B 606 (2001) 518 [Erratum ibid. B 790 (2008) 336] [hep-ph/0012052] [SPIRES];

J. Pradler and F.D. Steffen, Thermal gravitino production and collider tests of leptogenesis, Phys. Rev. D 75 (2007) 023509 [hep-ph/0608344] [SPIRES].

[7] M. Fujii, M. Ibe and T. Yanagida, Upper bound on gluino mass from thermal leptogenesis, Phys. Lett. B 579 (2004) 6 [hep-ph/0310142] [SPIRES];

D.G. Cerdeno, K.-Y. Choi, K. Jedamzik, L. Roszkowski and R. Ruiz de Austri, Gravitino dark matter in the CMSSM with improved constraints from BBN, JCAP 06 (2006) 005 [hep-ph/0509275] [SPIRES];

J. Pradler and F.D. Steffen, Constraints on the reheating temperature in gravitino dark matter scenarios, Phys. Lett. B 648 (2007) 224 [hep-ph/0612291] [SPIRES].

[8] W. Buchmüller, L. Covi, K. Hamaguchi, A. Ibarra and T. Yanagida, Gravitino dark matter in R-parity breaking vacua, JHEP 03 (2007) 037 [hep-ph/0702184] [SPIRES].

[9] F. Takayama and M. Yamaguchi, Gravitino dark matter without R-parity, Phys. Lett. B 485 (2000) 388 [hep-ph/0005214] [SPIRES]. 
[10] G. Bertone, W. Buchmüller, L. Covi and A. Ibarra, Gamma-rays from decaying dark matter, JCAP 11 (2007) 003 [arXiv:0709.2299] [SPIRES].

[11] A. Ibarra and D. Tran, Gamma ray spectrum from gravitino dark matter decay, Phys. Rev. Lett. 100 (2008) 061301 [arXiv:0709.4593] [SPIRES].

[12] A. Ibarra and D. Tran, Antimatter signatures of gravitino dark matter decay, JCAP 07 (2008) 002 [arXiv: 0804.4596] [SPIRES].

[13] K. Ishiwata, S. Matsumoto and T. Moroi, High energy cosmic rays from the decay of gravitino dark matter, arXiv:0805.1133 [SPIRES].

[14] L.J. Hall and M. Suzuki, Explicit R-parity breaking in supersymmetric models, Nucl. Phys. B 231 (1984) 419 [SPIRES].

[15] B.A. Campbell, S. Davidson, J.R. Ellis and K.A. Olive, Cosmological baryon asymmetry constraints on extensions of the standard model,

Phys. Lett. B 256 (1991) 484 [SPIRES];

W. Fischler, G.F. Giudice, R.G. Leigh and S. Paban, Constraints on the baryogenesis scale from neutrino masses, Phys. Lett. B 258 (1991) 45 [SPIRES];

H.K. Dreiner and G.G. Ross, Sphaleron erasure of primordial baryogenesis, Nucl. Phys. B 410 (1993) 188 [hep-ph/9207221] [SPIRES].

[16] For a review of bilinear $R$-parity breaking see $\mathrm{R}$. Barbier et al., $R$-parity violating supersymmetry, Phys. Rept. 420 (2005) 1 [hep-ph/0406039] [SPIRES].

[17] S. Lola, P. Osland and A.R. Raklev, Radiative gravitino decays from R-parity violation, Phys. Lett. B 656 (2007) 83 [arXiv:0707.2510] [SPIRES].

[18] J. Wess and J. Bagger, Supersymmetry and supergravity, Princeton University Press, Princeton U.S.A. (1992);

For the detailed computation see also M. Grefe, Neutrino signals from gravitino dark matter with broken R-parity, DESY-THESIS-2008-043 [SPIRES].

[19] T. Sjöstrand, S. Mrenna and P. Skands, PYTHIA 6.4 physics and manual, JHEP 05 (2006) 026 [hep-ph/0603175] [SPIRES].

[20] J.F. Navarro, C.S. Frenk and S.D.M. White, The structure of cold dark matter halos, Astrophys. J. 462 (1996) 563 [astro-ph/9508025] [SPIRES].

[21] A. Strumia and F. Vissani, Neutrino masses and mixings and., hep-ph/0606054 [SPIRES].

[22] T. Schwetz, M. Tortola and J.W.F. Valle, Three-flavour neutrino oscillation update, New J. Phys. 10 (2008) 113011 [arXiv:0808.2016] [SPIRES].

[23] G. Battistoni et al., A 3-dimensional calculation of atmospheric neutrino flux, Astropart. Phys. 12 (2000) 315 [hep-ph/9907408] [SPIRES];

Updated tables of the data are given at http://www.mi.infn.it/ battist/neutrino.html.

[24] G. Battistoni, A. Ferrari, T. Montaruli and P.R. Sala, The FLUKA atmospheric neutrino flux calculation, Astropart. Phys. 19 (2003) 269 [Erratum ibid. 19 (2003) 291] [hep-ph/0207035] [SPIRES]. 
[25] L. Pasquali and M.H. Reno, Tau neutrino fluxes from atmospheric charm, Phys. Rev. D 59 (1999) 093003 [hep-ph/9811268] [SPIRES].

[26] G. Ingelman and M. Thunman, High energy neutrino production by cosmic ray interactions in the sun, Phys. Rev. D 54 (1996) 4385 [hep-ph/9604288] [SPIRES].

[27] H. Athar, F.-F. Lee and G.-L. Lin, Tau neutrino astronomy in GeV energies, Phys. Rev. D 71 (2005) 103008 [hep-ph/0407183] [SPIRES].

[28] Official NASA Fermi website, http://fermi.gsfc.nasa.gov.

[29] Super-Kamiokande collaboration, K. Abe et al., A measurement of atmospheric neutrino flux consistent with tau neutrino appearance, Phys. Rev. Lett. 97 (2006) 171801 [hep-ex/0607059] [SPIRES].

[30] T. Kato, Tau neutrino appearance via neutrino oscillations in atmospheric neutrinos, Ph.D. thesis (Advisor: Chang Kee Jung), SUNY, Stony Brook U.S.A. (2007) [SPIRES].

[31] OPERA: Oscillation Project with Emulsion-tRacking Apparatus, http://operaweb.lngs.infn.it.

[32] S. Palomares-Ruiz, Model-independent bound on the dark matter lifetime, Phys. Lett. B 665 (2008) 50 [arXiv:0712.1937] [SPIRES].

[33] P. Gondolo, G. Gelmini and S. Sarkar, Cosmic neutrinos from unstable relic particles, Nucl. Phys. B 392 (1993) 111 [hep-ph/9209236] [SPIRES].

[34] ICECuBE collaboration, Contributions to the $10^{\text {th }}$ International Conference on Topics in Astroparticle and Underground Physics (TAUP) 2007, Sendai Japan September 11-15 2007, arXiv:0712.3524 [SPIRES].

[35] ICECubE collaboration, D.F. Cowen, Tau neutrinos in IceCube, J. Phys. Conf. Ser. 60 (2007) 227 [SPIRES].

[36] PAMELA: A Payload for Antimatter Matter Exploration and Light-nuclei Astrophysics, http://pamela.roma2.infn.it;

For the latest preliminary results on the position fraction we refer to M. Boezio, PAMELA - an experiment searching for dark matter with cosmic antiparticles, talk at the IDM08 Conference, Stockholm Sweden August 18-22 2008. 